\title{
Effect of Adjuvant Chemotherapy after Complete Resection for Pathologic Stage IB Lung Adenocarcinoma in High-Risk Patients as Defined by a New Recurrence Risk Scoring Model
}

\author{
Hyo Joon Jang, $M D^{1}$ \\ Sukki Cho, MD, PhD ${ }^{1,2}$ \\ Kwhanmien Kim, MD, PhD',2 \\ Sanghoon Jheon, MD, $\mathrm{PhD}^{1,2}$ \\ Hee Chul Yang, MD ${ }^{3}$ \\ Dong Kwan Kim, MD, PhD
}

\begin{abstract}
Purpose
We conducted a retrospective analysis to determine if adjuvant chemotherapy prolongs overall survival in patients with pathologic stage IB lung adenocarcinoma who had undergone complete resection and were defined as high-risk by a newly developed recurrence risk scoring model.
\end{abstract}

\section{Materials and Methods}

Patients who underwent curative resection for stage IB lung adenocarcinoma were analyzed with a newly developed recurrence risk scoring model and divided into a low-risk group and a high-risk group. The patients in the high-risk group were retrospectively divided into two groups based on whether they underwent adjuvant chemotherapy or observation. Recurrence-free survival and overall survival were compared between these two groups.

\section{Results}

A total of 328 patients who underwent curative resection between 2000 and 2009 were included in this study, of whom 110 (34\%) received adjuvant chemotherapy and 218 (67\%) underwent observation without additional treatment. According to our risk model, 167 patients (51\%) were high-risk and 161 (49\%) were low-risk. The 5-year recurrence-free survival rates and overall survival were $84.4 \%$ and $91.5 \%$ in low-risk patients and $53.9 \%$ and $74.7 \%$ in high-risk patients ( $p<0.001$ ). In high-risk patients, the 5-year overall survival rates were $77 \%$ among patients who underwent observation and $87 \%$ among those who underwent adjuvant chemotherapy $(p=0.019)$.

\section{Conclusion}

Adjuvant chemotherapy prolonged overall survival among high-risk patients who had undergone complete resection for stage IB lung adenocarcinoma.
Department of Thoracic and Cardiovascular

Surgery, Seoul National University Bundang

Hospital, 82 Gumi-ro 173beon-gil, Bundang-gu,

Seongnam 13620, Korea

Tel: 82-31-787-7132

Fax: 82-31-787-4050

E-mail: skcho@snubh.org

Received July 14, 2016

Accepted December 8, 2016

Published Online January 18, 2017
Key words

Adjuvant chemotherapy, Stage IB, Adenocarcinoma, High risk

\section{Introduction}

Lung cancer is the leading cause of cancer death for men and women worldwide [1]. The 5-year survival rate of stage IB lung cancer is $54 \%$, which is significantly lower than that of stage IA cancer (73\%) and similar to that of stage IIA cancer $(50 \%)$ [2]. Several randomized controlled clinical trials have been performed to assess strategies for increasing the survival rate in resected stage IB lung cancer; however, they have not resulted in the improvements that were expected [3-5]. Nonetheless, the Cancer and Leukemia Group B 9633 study showed that adjuvant chemotherapy led to an increased survival rate in stage IB patients with tumors $>$ $4 \mathrm{~cm}$ [6]. Therefore, the National Comprehensive Cancer Network has issued guidelines defining high-risk patients with pathologic stage IB cancer, for whom adjuvant chemotherapy may be recommended [7].

Although there is no question that adjuvant chemotherapy should only be performed in high-risk patients with stage IB 
lung cancer, controversy exists regarding how to define the risk level of patients. We previously identified three major risk factors for postoperative recurrence and created a new risk model that can distinguish high- and low-risk patients who have undergone curative resection to treat pathologic stage I lung adenocarcinoma [8].

We suggest that if adjuvant chemotherapy leads to better results than observation in patients with pathologic stage IB lung adenocarcinoma who are classified as high-risk in the new recurrence risk scoring model, a multicenter prospective randomized controlled trial may be warranted.

Therefore, this study was conducted to perform a retrospective analysis of the results of adjuvant chemotherapy in high-risk patients who had undergone curative resection for stage IB lung adenocarcinoma.

\section{Materials and Methods}

\section{Patients}

The data used in this study were collected from the records of 1,071 patients who underwent curative resection for pathologic stage I lung adenocarcinoma between January 2000 and December 2009 at three different hospitals. We excluded patients who had received any therapy prior to surgery. Of the 1,071 patients identified for this study, $743(69 \%)$ had stage IA cancer and $328(31 \%)$ had stage IB cancer. This study was approved by the Institutional Review Board of Seoul National University Bundang Hospital. Patients' informed consent was waived due to the retrospective nature of the study design.

\section{Risk stratification}

A previous study performed by our lung cancer study group identified three independent predictors of recurrence [8]: pathologic tumor size (hazard ratio [HR], 1.03; 95\% confidence interval [CI], 1.02 to $1.05 ; \mathrm{p}<0.001)$, corrected standardized uptake value (c-SUV) (HR, 1.08; 95\% CI, 1.05 to 1.11; $\mathrm{p}<0.001$ ), and lymphovascular invasion (LVI) (HR, $1.65 ; 95 \% \mathrm{CI}, 1.17$ to $2.33, \mathrm{p}=0.004)$. Based on the results of the Cox proportional hazard model, the total score was calculated as: Total score $=[0.02238964 \times($ tumor size, $\mathrm{mm})+$ $0.5993035 \times(\mathrm{c}-S U V)-0.01006747 \times(\mathrm{c}-S U V-0.8)+3+0.01423332 \times$ (cSUV-3.2)+3-0.00416585×(c-SUV-9)+3+0.1980969 $\times($ LVI)] $\times 44.99556-28$; terms introduced by addition (+) obligatorily have a positive value. For terms introduced by subtraction, negative values of the variable in question result in the term being treated as zero. LVI is calculated as 1 (present) or 0 (absent).

Score values were obtained for all patients. We were able predict the probability of 1-year, 3-year, and 5-year recurrence-free survival (RFS) for each patient by applying the total score to a nomogram or a corresponding score table. We defined the high-risk group as patients with total scores belonging to the 70th percentile or above. The recurrence rate in the development cohort was 20\%-30\% in each institution, and the 5-year RFS rate of the top 30 percentile high-risk score group was 59\% (95\% CI, 0.54 to 0.66 ), indicating that the results resembled stage IIA rather than stage IB. The cutoff value of high-risk sore was 104 point in our risk model.

\section{Adjuvant chemotherapy}

No uniform indication of adjuvant chemotherapy was used for the patients analyzed in this study. Adjuvant chemotherapy was usually performed if patients had wellknown risk factors, such as large tumor size, poor differentiation, LVI, or visceral pleural invasion (VPI). Chemotherapeutic agents also varied across centers. Oral tegafur/uracil was administered for 1-1.5 years at a dose of $400 \mathrm{mg}$ per day. Platinum-based adjuvant chemotherapy was started within 4 to 8 weeks of surgical resection. Four cycles of chemotherapy involving cisplatin $\left(80 \mathrm{mg} / \mathrm{m}^{2}\right)$ with paclitaxel or vinorelbine were performed. Decisions about dose reduction or dose delay were made by the treating medical oncologist at the time of the scheduled dose using objective and subjective criteria.

Patients were followed up every 6 months for the first 2 years, and then every 8 months for an additional 3 years. Data analyzed in this study included patient's history, physical examination, chest radiograph, and tumor markers. A chest computed tomography (CT) scan was taken every 6 months for the first 2 years, then every 8 months. If recurrence was suspected, either through newly presenting symptoms or scheduled tests, integrated positron emission tomography CT (PET-CT) was performed.

\section{Statistical analysis}

The length of the overall survival (OS) was defined as the interval between the date of surgical resection and the date of either death or the last follow-up. The period of freedom from recurrence was defined as the interval between the date of surgical resection and the date of the first recurrence or the last follow-up. An observation was censored at the last follow-up session when the patient was alive with recurrence-free status or died without recurrence. If a new tumor developed during follow-up that had a cell type that differed from the previous lung cancer, this newly formed tumor was considered a metachronous double primary cancer, and not 
recurrence. Moreover, if multiple lesions of the same histologic type according to the criteria of Martini and Melamed [9] showed lack of common lymphatic carcinoma, were located in different lobes, originated from carcinoma in situ, and after more than 3 years of disease-free survival, they were not considered recurrence. The Student's $t$ test and analysis of variance (ANOVA) were used to compare the distribution of continuous data, and Fisher exact test or the chisquared test was used to compare the frequencies of categorical measures among groups. Five-year RFS rates were analyzed using the Kaplan-Meier method, and survival curves were generated. Multivariate analyses were performed using the Cox proportional hazard model. Statistical analyses were performed using SPSS ver. 18.0 (SPSS Inc., Chicago, IL). p-values of $<0.05$ were considered to indicate statistical significance.

\section{Results}

\section{Patients}

Pathological stage IB lung adenocarcinoma was found in 328 patients with a mean age of 63.1 years, of whom 161

Table 1. Clinical characteristics of low-risk and high-risk patients in stage IB lung adenocarcinoma

\begin{tabular}{|c|c|c|c|}
\hline Characteristic & Low-risk group & High-risk group & p-value \\
\hline \multicolumn{4}{|l|}{ Sex } \\
\hline Male & $62(38.5)$ & $99(61.5)$ & $<0.001$ \\
\hline Female & $99(59.3)$ & $68(40.7)$ & \\
\hline Age (yr) & $62.4 \pm 10.2$ & $63.7 \pm 10.4$ & 0.276 \\
\hline \multicolumn{4}{|l|}{ Smoking } \\
\hline Never & $113(60.1)$ & $76(39.9)$ & $<0.001$ \\
\hline Ever & $48(34.5)$ & $91(65.5)$ & \\
\hline CEA (ng/mL) & $3.4 \pm 6.3$ & $7.3 \pm 16.6$ & 0.013 \\
\hline c-SUV & $2.8 \pm 1.4$ & $8.5 \pm 4.8$ & $<0.001$ \\
\hline Total tumor size on CT (mm) & $29.0 \pm 12.7$ & $34.0 \pm 10.1$ & $<0.001$ \\
\hline Solid size on CT (mm) & $23.1 \pm 12.6$ & $31.3 \pm 10.9$ & $<0.001$ \\
\hline Total tumor size (mm) & $27.2 \pm 10.0$ & $35.1 \pm 7.0$ & $<0.001$ \\
\hline Invasive size $(\mathrm{mm})$ & $26.9 \pm 9.9$ & $34.8 \pm 7.2$ & $<0.001$ \\
\hline \multicolumn{4}{|l|}{ Visceral pleural invasion } \\
\hline No & $56(42.4)$ & $76(57.6)$ & 0.048 \\
\hline Yes & $105(53.6)$ & $91(46.4)$ & \\
\hline \multicolumn{4}{|l|}{ Lymphovascular invasion } \\
\hline No & $113(54.6)$ & $94(45.4)$ & 0.009 \\
\hline Yes & $48(39.7)$ & $73(60.3)$ & \\
\hline \multicolumn{4}{|l|}{ EGFR mutation } \\
\hline Wild type & $86(51.2)$ & $82(48.8)$ & 0.012 \\
\hline Mutation & $47(58.0)$ & $34(42.0)$ & \\
\hline \multicolumn{4}{|l|}{ Recurrence } \\
\hline No & $138(60.0)$ & $92(40.0)$ & $<0.001$ \\
\hline Yes & $23(23.5)$ & $75(76.5)$ & \\
\hline \multicolumn{4}{|l|}{ Survival } \\
\hline Alive & 148 (53.2) & $130(46.8)$ & $<0.001$ \\
\hline Dead & $13(26.0)$ & $37(74.0)$ & \\
\hline \multicolumn{4}{|l|}{ Adjuvant chemotherapy } \\
\hline No & $104(47.7)$ & $114(52.3)$ & 0.482 \\
\hline Yes & $57(51.8)$ & $53(48.2)$ & \\
\hline
\end{tabular}

Values are presented as number $(\%)$ or mean \pm standard deviation. CEA, carcinoembryonic antigen; c-SUV, corrected standardized uptake value; CT, computed tomography; EGFR, epidermal growth factor receptor. 


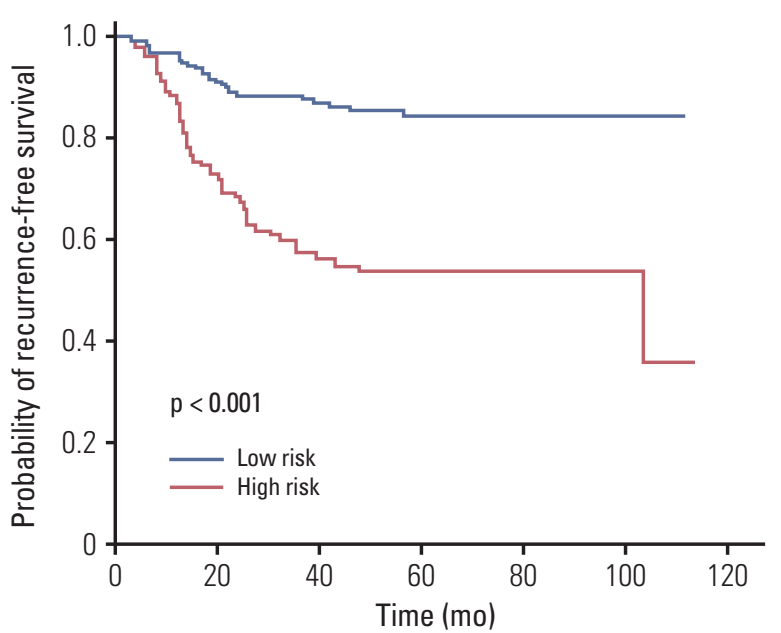

A

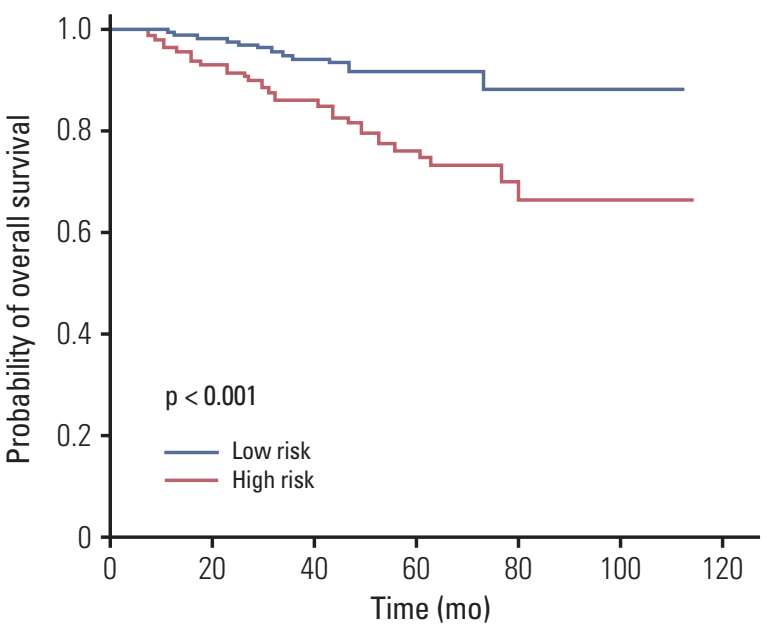

Fig. 1. Kaplan-Meier estimates of recurrence-free survival (A) and overall survival (B) between low-risk and high-risk patients. (A) The 5-year recurrence-free survival rates were $84.4 \%$ in low-risk patients and 53.9\% in high-risk patients. (B) The 5-year overall survival rates were $91.5 \%$ in low-risk patients and $74.7 \%$ in high-risk patients.

$(49 \%)$ were male and $167(51 \%)$ were female. There were 139 ever-smokers $(42 \%)$ and 189 never-smokers (58\%). All patients underwent preoperative PET-CT imaging, with a mean c-SUV value of 5.69. According to pathological reports, the mean total pathological and invasive sizes were $31.2 \mathrm{~mm}$ and $30.9 \mathrm{~mm}$, respectively. LVI was found in 121 patients $(37 \%)$ and VPI was found in 196 patients (60\%). A total of 110 patients $(34 \%)$ received adjuvant chemotherapy.

\section{Risk stratification}

Overall, 167 patients (51\%) were classified as high-risk and 161 patients $(49 \%)$ as low-risk according to risk calculation based on the nomogram derived from our previously reported recurrence risk model. The characteristics of the two groups are shown in Table 1 . The high-risk patients were more likely to be male and ever-smokers than the low-risk patients. Male patients had a significantly higher SUVmax (6.6 vs. $4.9, \mathrm{p}=0.001$ ) than female patients; however, there was no difference in age, performance status, tumor size, or the presence of LVI, VPI, and comorbidity. The high-risk patients had higher carcinoembryonic antigen levels, higher corrected standard uptake values based on PET-CT imaging, and larger total or invasive tumor size. Poor differentiation, VPI, LVI, and wild-type epidermal growth factor receptor (EGFR) were found more frequently in high-risk patients.

\section{Survival}

The mean follow-up period was 49.3 months (range, 6 to
106 months). Recurrence was documented in 98 patients $(30 \%)$, including 23 patients $(14 \%)$ in the low-risk group and $75(45 \%)$ in the high-risk group $(\mathrm{p}<0.001)$. Fifty patients $(15 \%)$ died during follow-up period: $13(8 \%)$ in the low-risk group and $37(22 \%)$ in the high-risk group. The Kaplan-Meier estimates of RFS and OS are presented in Fig. 1. The 5-year RFS and OS rates were $84.4 \%$ and $91.5 \%$ in low-risk patients and $53.9 \%$ and $74.7 \%$ in high-risk patients, respectively, which was a significant difference.

\section{Adjuvant chemotherapy}

A total of 110 patients received adjuvant chemotherapy, including 57 low-risk and 53 of high-risk patients. Although patients who received adjuvant chemotherapy were significantly younger, Eastern Cooperative Oncology Group performance status and presence of comorbidity were not significantly different between patients with observation and adjuvant chemotherapy (Table 2). EGFR mutation analysis was performed in $64 \%$ of patients with observation, while it was performed in $100 \%$ of patients with chemotherapy. The prevalence of EGFR mutation was significantly higher in patients with chemotherapy.

In addition to cisplatin, the regimens of platinum-based doublet chemotherapy included vinorelbine in 17, gemcitabine in 14, and paclitaxel in eight patients. The regimen of gemcitabine and oxaliplatin was used in 10 patients. The 5 -year OS rate was 79\% in the observation group and $92 \%$ in the chemotherapy group, which was a significant difference ( $p=0.001$ ) (Fig. 2). The 5-year RFS rate was $64 \%$ in the obser- 
Table 2. Comparison of clinical characteristics between patients with observation and chemotherapy in stage IB lung adenocarcinoma

\begin{tabular}{|c|c|c|c|}
\hline Characteristic & Observation $(\mathrm{n}=\mathbf{2 1 8})$ & Chemotherapy (n=110) & p-value \\
\hline \multicolumn{4}{|l|}{ Sex } \\
\hline Male & $106(51.4)$ & $55(50.0)$ & 0.814 \\
\hline Female & $112(48.6)$ & $55(50.0)$ & \\
\hline Age $(y r)$ & $64.8 \pm 10.8$ & $60.4 \pm 8.7$ & 0.001 \\
\hline \multicolumn{4}{|l|}{ Smoking } \\
\hline Never & $125(57.3)$ & $64(58.2)$ & 0.369 \\
\hline Ever & $93(42.7)$ & $46(41.8)$ & \\
\hline \multicolumn{4}{|c|}{ ECOG performance status } \\
\hline $0-1$ & $201(92.6)$ & $103(93.6)$ & 0.120 \\
\hline $2-4$ & $17(7.4)$ & $7(6.4)$ & \\
\hline \multicolumn{4}{|l|}{ Comorbiditya) } \\
\hline None & $119(54.6)$ & $62(56.4)$ & 0.852 \\
\hline Presence & $99(45.4)$ & $48(43.6)$ & \\
\hline \multicolumn{4}{|l|}{ EGFR mutation } \\
\hline Wild type & $96(44.0)$ & $72(65.5)$ & 0.001 \\
\hline Mutation & $43(19.7)$ & $38(34.5)$ & \\
\hline \multicolumn{4}{|l|}{ Recurrence } \\
\hline No & $147(67.4)$ & $83(75.5)$ & 0.134 \\
\hline Yes & $71(32.6)$ & $27(24.5)$ & \\
\hline
\end{tabular}

Values are presented as number (\%) or mean \pm standard deviation. ECOG, Eastern Cooperative Oncology Group; EGFR, epidermal growth factor receptor. ${ }^{a}$ Comorbidity=chronic obstructive pulmonary disease, diabetic mellitus, hypertension, cerebrovascular attack, cardiovascular disease other than hypertension.

vation group and $76 \%$ in the chemotherapy group, which was a marginally significant difference $(\mathrm{p}=0.053)$.

In low-risk patients, the 5 -year OS rate was $89 \%$ with observation and $96 \%$ with chemotherapy, which was significantly different $(\mathrm{p}=0.032)$. In high-risk patients, the 5-year OS rate was $69 \%$ with observation and $87 \%$ with chemotherapy, which was also significantly different ( $\mathrm{p}=0.019)$ (Fig. 3). Among 53 high-risk patients, oral agents were administered to 30 patients and intravenous chemotherapeutic agents (IV chemotherapy) to 23 . When 23 patients (IV chemotherapy) were compared with 114 patients (observation) in the highrisk group, mean survival time was 84 months and 76 months in patients with IV chemotherapy and observation, respectively, which was significantly longer in patients with IV chemotherapy ( $\mathrm{p}=0.049$ ).

\section{Complications of adjuvant chemotherapy}

There was no discontinuation in patients who received oral agents as adjuvant chemotherapy; however, four patients suffered from gastrointestinal (GI) problems and two from internal bleeding. In patients who received IV chemotherapeutic agents, the discontinuation rate was $10 \%$ and complications (all GI trouble) developed in three patients.

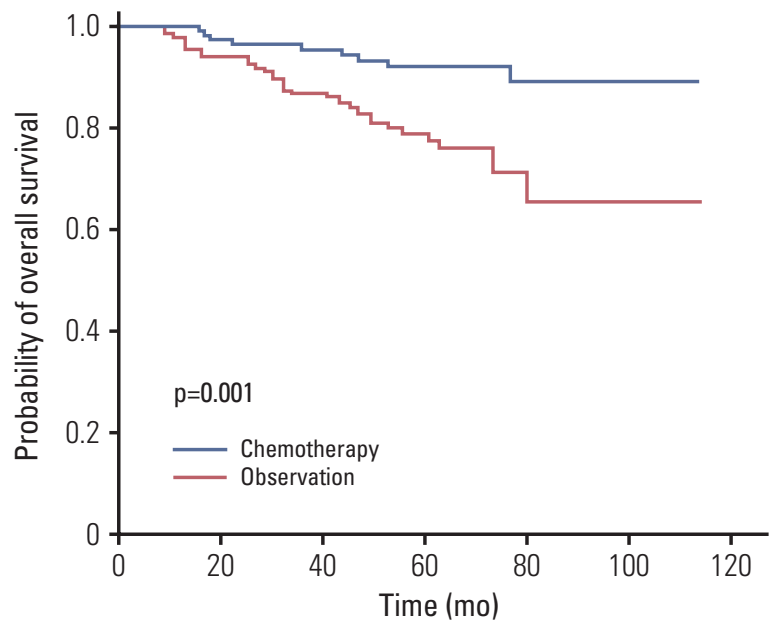

Fig. 2. Kaplan-Meier estimates of overall survival between observation and chemotherapy. The 5-year overall survival rate was $79 \%$ in patients who received observation and $92 \%$ in those who received chemotherapy. 


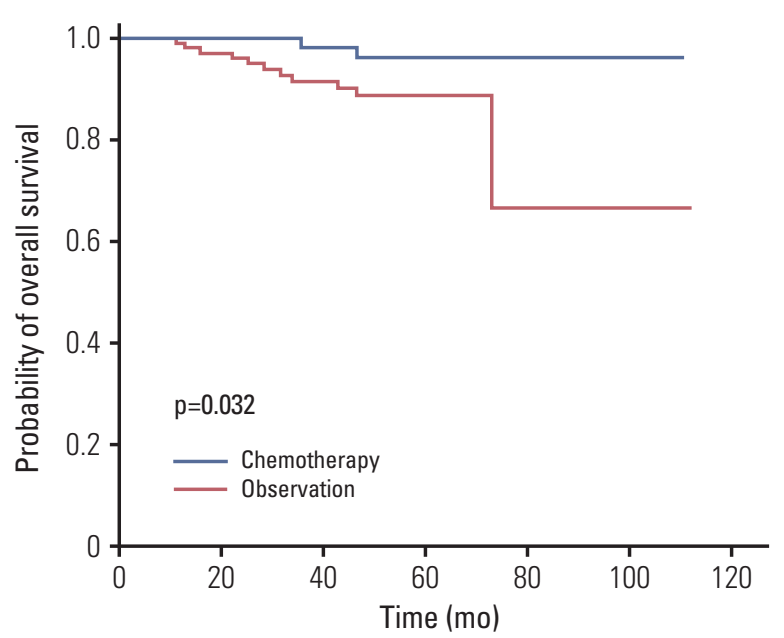

A

Fig. 3. Kaplan-Meier estimates of overall survival between observation and chemotherapy in low-risk (A) and high-risk (B) patients. (A) The 5-year overall survival rate was $89 \%$ in patients with observation and $96 \%$ for those who received chemotherapy in high-risk patients. (B) The 5-year overall survival rate was $77 \%$ in patients who received observation and $87 \%$ in those who received chemotherapy in high-risk patients.

\section{Discussion}

Two major conclusions can be drawn from this retrospective study. First, the nomogram of our newly developed risk model appropriately defined risk groups for recurrence, because the high-risk group had a significantly lower 3-year disease-free survival rate than low-risk patients. Second, adjuvant chemotherapy significantly prolonged the 5-year OS rate of high-risk patients when compared to observation alone.

Traditionally, lung cancer has been considered to be an aggressive cancer, with a total survival rate of less than $20 \%$. To increase the survival rate, multi-modality therapies have been developed, including adjuvant chemotherapy, adjuvant chemoradiation, and neoadjuvant therapy, with very encouraging results. Therefore, adjuvant chemotherapy is routinely performed for pathologic stage II non-small cell lung cancer (NSCLC), while adjuvant chemotherapy and radiotherapy are routinely performed for pathologic stage III NSCLC $[3-5,10]$. However, the effectiveness of adjuvant therapy for completely resected stage I NSCLC is controversial, especially for stage IB NSCLC. For stage IA tumors, studies have consistently shown that adjuvant chemotherapy has a negative effect on OS [11]. This is because, although adjuvant chemotherapy can decrease the recurrence rate after curative resection, the toxicity of the chemotherapeutic agents and immunosuppression can increase treatment-related mortality. The Cancer and Leukemia Group B 9633 study enrolled
200 patients with pathologic stage IB cancer and found in their preliminary report that adjuvant chemotherapy was associated with an HR for overall mortality of 0.62 (0.44-0.89, $\mathrm{p}<0.014$ ), indicating that adjuvant chemotherapy was beneficial in these patients [6]. Although the results that were updated 4 years later no longer showed a significant effect on OS, adjuvant chemotherapy consistently led to an improved survival rate in patients with tumors larger than 4 $\mathrm{cm}$. Therefore, the National Comprehensive Cancer Network guidelines define poorly differentiated tumors, vascular invasion, wedge resection, tumor size $>4 \mathrm{~cm}$, VPI, and incomplete lymph node sampling as high-risk factors in surgically resected IB NSCLC, and recommend adjuvant chemotherapy to patients with these risk factors [7]. However, these risk factors have not been shown to be independent indications for adjuvant chemotherapy for patients with stage IB NSCLC. The lack of a widely accepted and clear definition of high-risk patients has led to inconsistent clinical application of adjuvant chemotherapy. Therefore, in our previous study, clinical, radiopathological, and outcome data were evaluated for 1,700 patients with pathologic stage I adenocarcinoma treated at four different centers. A nomogram for recurrence prediction was developed using Cox proportional hazards regression, and this model was validated in a cohort of 460 patients in two other hospitals. Pathologic tumor size, c-SUV, and LVI were identified as independent predictors for recurrence [8].

Our previous study led us to the conclusion that it is extremely important to develop guidelines to determine 
which high-risk patients, as defined by our new nomogram, should be given adjuvant chemotherapy for stage IB adenocarcinoma. Therefore, before embarking on a multicenter prospective randomized controlled trial, it was important to validate the role of adjuvant chemotherapy in patients with high-risk stage IB adenocarcinoma.

In this study, the OS advantage at 5 years was found to be $10 \%$ in high-risk patients who were treated with chemotherapy. However, these results should be interpreted with caution because of the inconsistent indications for chemotherapy and the use of various chemotherapeutic agents and cycles. A particular weak point is the lack of uniform indications for adjuvant chemotherapy and the variety of chemotherapeutic agents in each center.

Another point to consider is that chemotherapy also led to improved outcomes in low-risk patients, as defined by our new model. In low-risk patients, the 5-year OS rate was $96 \%$ with chemotherapy and $89 \%$ with observation. Although the difference was smaller than that found in the high-risk group, it was also statistically significant. There are multiple reasons for which chemotherapy also improved the OS time of the low-risk group. First, although smaller tumors were found in the low-risk group, the average total size was 2.7 $\mathrm{cm}$, which still qualifies as a T1b tumor. Second, and more importantly, the new risk model did not take VPI into account as a risk factor. VPI is known to be a major determinant of tumor staging and a risk factor for recurrence and poor survival [12]. However, in our previous study, VPI was not found to be an independent prognostic factor, and VPI was therefore rather common in the low-risk group. Moreover, disagreement exists about the prognostic role of VPI. Mizuno et al. [13] showed that stage IB adenocarcinoma patients without pleural invasion had a rate of favorable outcomes that was almost the same as that of stage IA patients, and therefore suggested that adjuvant chemotherapy can be omitted for stage IB adenocarcinoma without pleural invasion. In contrast, Nitadori et al. [14] showed that VPI did not affect the recurrence or OS among patients with lung adenocarcinoma when tumors were smaller than $2 \mathrm{~cm}$. In this study, patients with stage IB adenocarcinoma and VPI had a lower 5-year OS time than those without VPI; however, no difference in the 5 -year OS rate was found between patients with VPI who were treated with adjuvant chemotherapy and those who underwent observation. Tumor size, SUVmax, and presence of LVI were prognostic factors in the new recurrence risk scoring model of this study. Because male patients had slightly larger tumor and significantly higher SUVmax, significantly more male patients were included in the high-risk group.

In patients with adjuvant chemotherapy, OS was significantly longer; however, RFS was not significantly different between the two groups. There might be a different pattern of each group. Therefore, even after recurrence, patients with adjuvant chemotherapy might have relatively treatable recurrence when compared with patients who only underwent observation. Accordingly, even though RFS was minimally different, the OS was significantly different.

It should be noted that this study had several limitations. First, although this was a large-scale, multicenter study, it was based on retrospective data; therefore, these results cannot be generalized because different results might be shown in different study populations. To overcome this limitation, another validation group or prospective randomized trial would be needed. Second, we could not classify death into cancer-related death, treatment-related death, or unrelated death; accordingly, better OS of patients with chemotherapy may result from better conditions such as younger age, better performance status, and less comorbidity. Third, EGFR mutation analysis was only performed in $64 \%$ of patients who received observation. Because the study population was enrolled from three different hospitals in this study, EGFR mutation was not evaluated in all of the patients who were designated as the observation group. This difference may have influenced the better survival observed for patients who received adjuvant chemotherapy.

This retrospective study demonstrated that adjuvant chemotherapy had a positive effect on the OS rate of patients who had undergone curative resection for stage IB adenocarcinoma and were identified as high-risk by our new risk model. In light of these results, it may be worthwhile to conduct a multicenter prospective randomized trial to evaluate the effects of chemotherapy on such patients.

\section{Conflicts of Interest}

Conflict of interest relevant to this article was not reported.

\section{Acknowledgments}

This work was supported by the R\&D program of the Korean Ministry of Knowledge and Economy/Korea Evaluation Institute of Industrial Technology (MKE/KEIT) (10040393; Development and commercialization of molecular diagnostic technologies for lung cancer through clinical validation) and the Korean Foundation for Cancer Research (CB-2011-02-01). 


\section{References}

1. Jemal A, Bray F, Center MM, Ferlay J, Ward E, Forman D. Global cancer statistics. CA Cancer J Clin. 2011;61:69-90.

2. Goldstraw P, Crowley J, Chansky K, Giroux DJ, Groome PA, Rami-Porta R, et al. The IASLC Lung Cancer Staging Project: proposals for the revision of the TNM stage groupings in the forthcoming (seventh) edition of the TNM Classification of malignant tumours. J Thorac Oncol. 2007;2:706-14.

3. Arriagada R, Bergman B, Dunant A, Le Chevalier T, Pignon JP, Vansteenkiste J, et al. Cisplatin-based adjuvant chemotherapy in patients with completely resected non-small-cell lung cancer. N Engl J Med. 2004;350:351-60.

4. Douillard JY, Rosell R, De Lena M, Carpagnano F, Ramlau R, Gonzales-Larriba JL, et al. Adjuvant vinorelbine plus cisplatin versus observation in patients with completely resected stage IB-IIIA non-small-cell lung cancer (Adjuvant Navelbine International Trialist Association [ANITA]): a randomised controlled trial. Lancet Oncol. 2006;7:719-27.

5. Winton T, Livingston R, Johnson D, Rigas J, Johnston M, Butts $\mathrm{C}$, et al. Vinorelbine plus cisplatin vs. observation in resected non-small-cell lung cancer. N Engl J Med. 2005;352:2589-97.

6. Strauss GM, Herndon JE 2nd, Maddaus MA, Johnstone DW, Johnson EA, Harpole DH, et al. Adjuvant paclitaxel plus carboplatin compared with observation in stage IB non-small-cell lung cancer: CALGB 9633 with the Cancer and Leukemia Group B, Radiation Therapy Oncology Group, and North Central Cancer Treatment Group Study Groups. J Clin Oncol. 2008;26:5043-51.

7. Carlson RW, Larsen JK, McClure J, Fitzgerald CL, Venook AP, Benson AB 3rd, et al. International adaptations of NCCN clin- ical practice guidelines in oncology. J Natl Compr Canc Netw. 2014;12:643-8.

8. Yang HC, Kim HR, Jheon S, Kim K, Cho S, Ahn S, et al. Recurrence risk-scoring model for stage I adenocarcinoma of the lung. Ann Surg Oncol. 2015;22:4089-97.

9. Martini N, Melamed MR. Multiple primary lung cancers. J Thorac Cardiovascular Surg. 1975;70:606-12.

10. Ramnath N, Dilling TJ, Harris LJ, Kim AW, Michaud GC, Balekian AA, et al. Treatment of stage III non-small cell lung cancer: diagnosis and management of lung cancer, 3rd ed: American College of Chest Physicians evidence-based clinical practice guidelines. Chest. 2013;143(5 Suppl):e314S-40S.

11. Scagliotti GV, Fossati R, Torri V, Crino L, Giaccone G, Silvano $\mathrm{G}$, et al. Randomized study of adjuvant chemotherapy for completely resected stage I, II, or IIIA non-small-cell lung cancer. J Natl Cancer Inst. 2003;95:1453-61.

12. Shimizu K, Yoshida J, Nagai K, Nishimura M, Ishii G, Morishita $Y$, et al. Visceral pleural invasion is an invasive and aggressive indicator of non-small cell lung cancer. J Thorac Cardiovasc Surg. 2005;130:160-5.

13. Mizuno T, Ishii G, Nagai K, Yoshida J, Nishimura M, Mochizuki T, et al. Identification of a low risk subgroup of stage IB lung adenocarcinoma patients. Lung Cancer. 2008;62: 302-8.

14. Nitadori J, Colovos C, Kadota K, Sima CS, Sarkaria IS, Rizk $\mathrm{NP}$, et al. Visceral pleural invasion does not affect recurrence or overall survival among patients with lung adenocarcinoma $\leq 2 \mathrm{~cm}$ : a proposal to reclassify T1 lung adenocarcinoma. Chest. 2013;144:1622-31. 